\title{
Changing presentation of prostate cancer in a UK population - 10 year trends in prostate cancer risk profiles in the East of England
}

\author{
D C Greenberg ${ }^{1}$, K A Wright ${ }^{1}$, A Lophathanon ${ }^{2,3}$, K R Muir ${ }^{3}$ and V J Gnanapragasam ${ }^{\star, 4,5}$
}

${ }^{1}$ Public Health England, National Cancer Registration Service (Eastern Office), Cambridge CB22 3AD, UK; ${ }^{2}$ Division of Health Sciences, Warwick Medical School, University of Warwick, Coventry CV4 7AL, UK; ${ }^{3}$ Institute of Population Health, University of Manchester, Manchester M13 9PL, UK; ${ }^{4}$ Translational Prostate Cancer Group, Hutchison/MRC Research centre, University of Cambridge, Cambridge CB1 OXZ, UK and ${ }^{5}$ Department of Urology, Addenbrookes Hospital, Hills Road, Cambridge CB2 OXZ, UK

Background: Prostate cancer incidence is rising in the United Kingdom but there is little data on whether the disease profile is changing. To address this, we interrogated a regional cancer registry for temporal changes in presenting disease characteristics.

Methods: Prostate cancers diagnosed from 2000 to 2010 in the Anglian Cancer Network $(n=21$ 044) were analysed. Risk groups (localised disease) were assigned based on NICE criteria. Age standardised incidence rates (IRs) were compared between 2000-2005 and 2006-2010 and plotted for yearly trends.

Results: Over the decade, overall IR increased significantly $(P<0.00001)$, whereas metastasis rates fell $(P<0.0007)$. For localised disease, IR across all risk groups also increased but at different rates $(P<0.00001)$. The most striking change was a three-fold increase in intermediate-risk cancers. Increased IR was evident across all PSA and stage ranges but with no upward PSA or stage shift. In contrast, IR of histological diagnosis of low-grade cancers fell over the decade, whereas intermediate and high-grade diagnosis increased significantly $(P<0.00001)$.

Conclusion: This study suggests evidence of a significant upward migration in intermediate and high-grade histological diagnosis over the decade. This is most likely to be due to a change in histological reporting of diagnostic prostate biopsies. On the basis of this data, increasing proportions of newly diagnosed cancers will be considered eligible for radical treatment, which will have an impact on health resource planning and provision.

Prostate cancer is the leading male cancer in the United Kingdom and a principal cause of cancer-related mortality (http://www.info. cancerresearchuk.org/cancerstats/types/prostate/). As in many countries, the United Kingdom has no organised screening programme and this is unlikely to be introduced in the near future; at least not based on the serum PSA test (Neal et al, 2009). Instead, the diagnosis of prostate cancer in the United Kingdom is based on selective testing and referral by primary care physicians using the Department of Health guidelines (Department of Health. The NHS cancer plan. London: Department of Health, 2000; National Institute for Health and Clinical Excellence, 2005).
Referral guidelines for suspected cancer. Clinical guideline 27). Despite the lack of screening, it is clear that the numbers of cases diagnosed annually in the United Kingdom is rising and is set to increase further in future years (Mistry et al, 2011). PSA-based screening is known to detect many indolent low-risk cancers that may not need active treatment (Schröder et al, 2009). Equally, there is evidence that the numbers of men diagnosed with metastatic disease is reduced as a result of screening (Schröder et al, 2009). In contrast, there are no data on the risk profile of cancers detected through primary care-based assessments and referrals to prostate diagnostic clinics. In particular, there is no study that has 
investigated whether UK prostate cancer risk profiles have remained similar or changed over time.

The risk profile of a tumour is the most important element in deciding how a man with prostate cancer is managed. In men with localised non-metastatic disease, risk is defined by the presenting PSA, tumour stage and histological grade and stratified as low, intermediate or high risk. The specific criteria are defined in the UK National Institute for Clinical Excellence guidelines (due to be updated in the next year) (http://www.guidance.nice.org.uk/ CG58/Guidance/pdf/English). Understanding the nature and types of cancers diagnosed in a health system is crucial for early detection of demographic changes in disease presentation, planning of resource needs and the impact on delivery of clinical services. It can also inform better targeting of populations that will benefit from early detection and intervention. In this study, we interrogated a UK regional cancer registry for patterns of changes in presentation of prostate cancer over the last 10 years. Our primary goal was to look for any evidence of changes in presenting risk profiles of prostate cancers diagnosed across this period.

\section{MATERIALS AND METHODS}

Patient cohort. Prostate cancers (ICD10 site: C61) diagnosed in residents of the Anglia Cancer Network area in the East of England region between 2000 and 2010 were registered by the National Cancer Registration Service - Eastern Office (NCRS(E)). This area covers $\sim 2.67$ million people (1.32 million male) in northern and central Bedfordshire, Cambridgeshire, Norfolk, Peterborough and Suffolk. Primary sources of information include electronic and paper-based reports and clinical notes from hospitals and pathology laboratories. Data elements recorded by NCRS(E) include age, hospitals of diagnosis and treatment, tumour site, morphology, TNM (fifth edition up to 2009 and seventh edition in 2010) stage and stage components, Gleason grade, and PSA. The stage assigned to each tumour is an integrated TNM stage at diagnosis; this assignment was made by the NCRS(E) consultant oncologist and/or consultant histopathologist based on combined clinical, imaging and pathological information. Recent reports have highlighted the completeness of staging information at NCRS(E) (Department of Health, Delivering the Cancer Reform Strategy. Report by the Controller and Auditor General, http://www.nao.org. uk/wp-content/uploads/2010/11/1011568.pdf). A total of 21044 cases were identified, of whom 19323 (91.8\%) were diagnosed at one of the main regional NHS trusts (Norfolk and Norwich, Cambridge, Ipswich, West Suffolk, James Paget, QE King's Lynn, Peterborough, Bedford and Hinchingbrooke; a further 821 (3.9\%) were diagnosed at one of the eight private hospitals in the network area. The remaining 900 cases $(4.3 \%)$ were diagnosed at hospitals outside the area or at hospices or by GPs. Overall, 18111 (86.1\%) of the diagnoses were microscopically verified, 1966 (9.3\%) were diagnosed by imaging, 816 (3.9\%) were clinical diagnoses and the remaining $151(0.7 \%)$ cases were diagnosed by data from death certificates. Analysis was done for the whole cohort and then stratified by age: $<60$ years, $60-69$ years, $70-79$ years and $>80$ years. The number of men with metastasis or localised disease was available by year and by age groups. For localised prostate cancer (non-metastatic), cases were further divided into risk subgroups based on the NICE guidelines criteria for PSA, grade and stage and stratified as low, intermediate or high risk (http:// www.guidance.nice.org.uk/CG58/Guidance/pdf/English).

Statistical analysis. Annual incidence rates (IRs) per 100000 population were calculated for overall and age specific cohorts. For localised prostate cancer, yearly IRs of each risk group for the whole cohort and stratified by age groups were also computed. Overall and age-specific incidence curves were then plotted for visual trends. The statistical test for IR change over two time periods (2000-2005 and 2006-2010) were assessed. To compute IRs, we summed cases and population between 2000-2005 and 2006-2010 and then calculated 5-year rates. This was done for incidence, metastasis and for localised disease, each risk group as well as the subcomponents of PSA, stage and histological grade. Difference in IR ratio between the two periods, the s.e. and confidence interval for IR were calculated using EpiBasic software developed by Department of Public Health, Institute for Folkesundhed, Denmark, which is available at http://www. folkesundhed.au.dk/uddannelse/software. A significant difference was considered when the $P$-value $<0.05$.

\section{RESULTS}

Incidence and presentation of prostate cancer. Analysis of overall prostate cancer IRs demonstrated a steady increase in numbers detected over the last 10 years by nearly $40 \%$ (Figure 1 ). To investigate this further, the IR in 2000-2005 and 2006-2010 were compared (Table 1). Here there was a significant increase in IR between the two time periods (325 vs 495, $P<0.00001$ ). In contrast, in the same periods, IR of the proportions of men presenting with metastatic disease actually fell (58 vs 51, $P=0.0007)$. Analysis by age groups identified that the increase in incidence was most pronounced in men aged under 60 years (over two-fold; Figure 2). These data corroborate the notion of an increase in prostate cancer incidence in the UK populations. We have observed the greatest increase in IR amongst younger men. In contrast, presentation with metastatic disease appears to be falling in the same cohorts over time.

Risk profiles of cancers diagnosed with non-metastatic disease. A key aim of the study was to investigate for any changes in the risk categorisation of localised cancers diagnosed in the last decade. To assess this, non-metastatic cancers were risk categorised based on the NICE criteria by Gleason grade, stage and presenting PSA. In comparison of the first and second half of the decades, there was a significant increase in IR across all risk groups albeit at different rates $(P<0.00001$; Table 1; Figure 3$)$. The most striking change was observed in the proportions of intermediate-risk cancers where there was a greater than three-fold overall increase in IR observed. In contrast, low-risk cancers increased at a much more modest rate. Analysis by age groups demonstrated that this proportional increase in intermediate-risk disease was apparent across all age groups, for example, four-fold in men aged $\leqslant 60$ years and fivefold in men aged 60-69 years (Figure 3). High-risk cancer IR were also increased across most age groups but at a more gradual pace

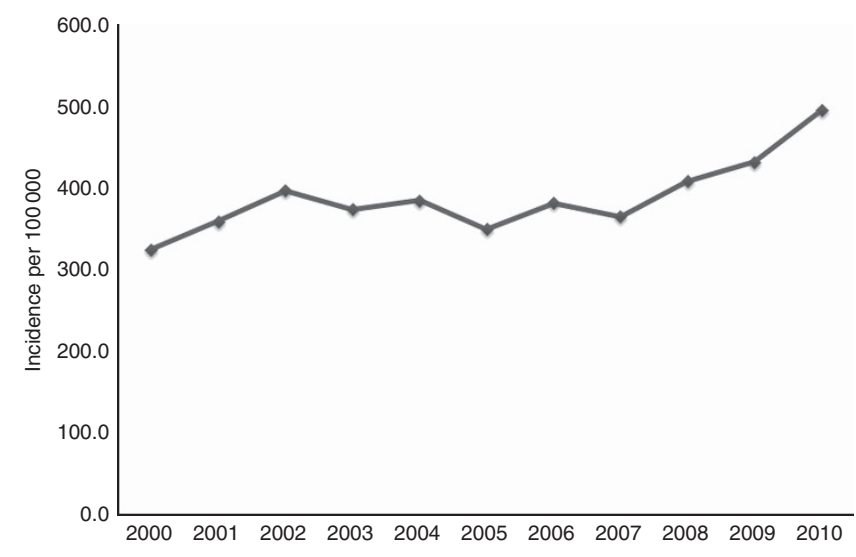

Figure 1. Incidence rate of prostate cancer in the Anglian Cancer Network 2000-2010. 
Table 1. Incidence rate (IR) changes in prostate cancer diagnostic characteristic between 2000-2005 and 2006-2010

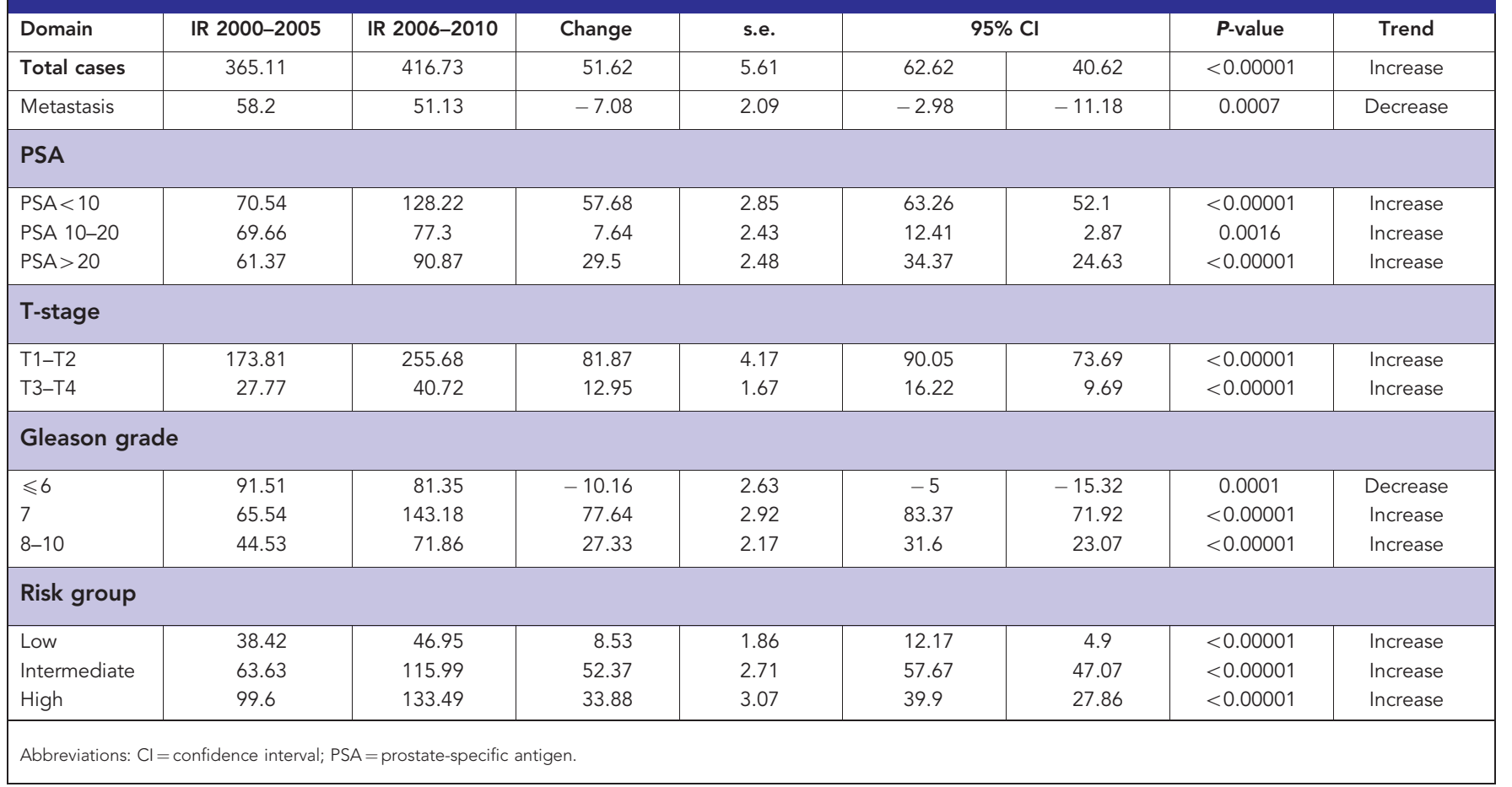

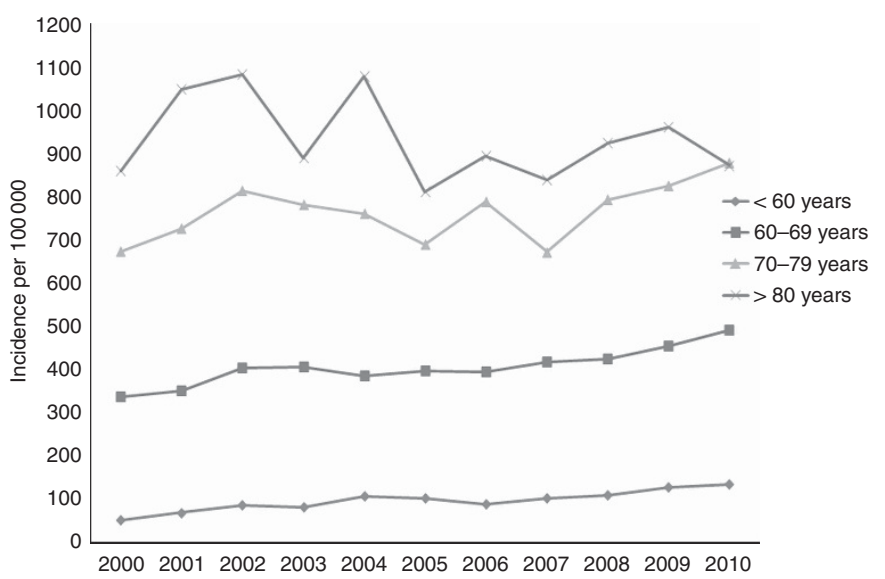

Figure 2. Incidence rate of prostate cancer stratified by age groups in the Anglia Cancer Network 2000-2010.

(three-fold in men aged $<60$ years and two-fold in men aged 60-69 and 70-79 years; Figure 3). There was however very little change amongst men aged over 80 years. These results suggest that although there has been an increase across all risk groups over the last decade, intermediate-risk cancers are now the most common cancer group diagnosed. To better understand the underlying basis of this change, we next analysed each subcomponent of the risk group attributes separately.

Changes in presenting PSA. Presentation in the three risk definitions for PSA were compared $(<10,10-20$ and $>20 \mathrm{ng} \mathrm{ml}^{-1}$ ). In analysis of the whole group, there was a statistically significant increase in the rates of men presenting in all three categories between the two time periods of 2000-2005 and 2006-2010 (Table 1). Analysis of the yearly data showed that the
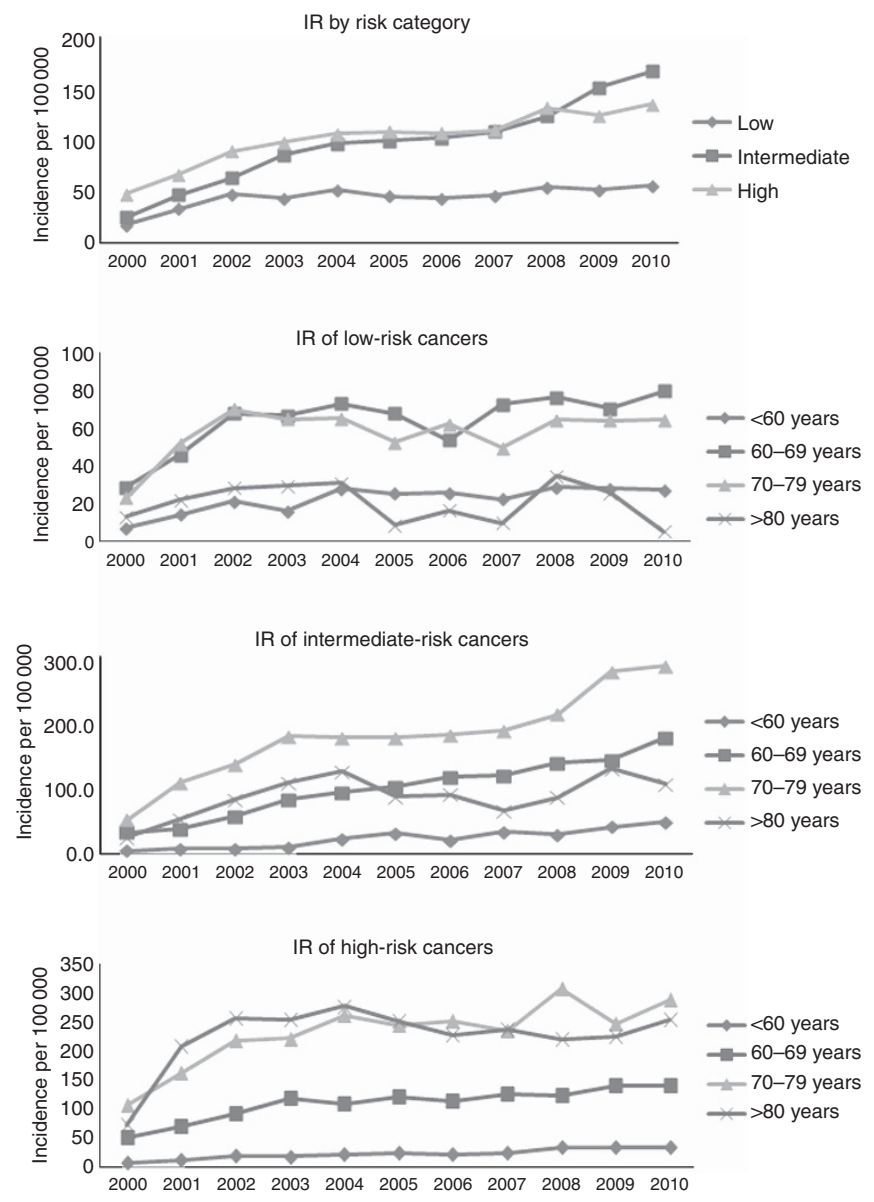

Figure 3 . Incidence rates (IR) stratified by risk group and by age group 2000-2010. 
most dramatic increase was actually seen in the relative numbers of new diagnosis with a PSA of $<10 \mathrm{ng} \mathrm{ml}^{-1}$ (low-risk group; Figure 4). Here, there was a six-fold increase in IRs between 2000 and 2010. In contrast, presentation with a PSA of $10-20 \mathrm{ng} \mathrm{ml}^{-1}$ and $>20 \mathrm{ng} \mathrm{ml}^{-1}$ increased by only two- to three-fold during this period.

Changes in stage presentation. Stage is arguably the most unreliable of the parameters used to ascribe risk in prostate cancer in diagnosis records. In cancer registry data, it is based on both pathological stage and/or on clinical and imaging assessment. For this subanalysis, stage data were simplified and dichotomised as either T1-T2 (organ confined) or T3-T4 (locally advanced). Analysis of the whole cohort revealed a significant increase in IR of both groups across the two time periods: T1-T2 IR 174 vs 256 $(P<0.00001)$, T3-T4 IR 27 vs $40(P<0.00001$; Table 1$)$. Notably, the relative proportions of men who were organ confined and locally advanced in both time periods, however, were very similar suggesting that there has not been a significant shift to detection of more organ-confined disease over the decade. Analysis of the yearly data similarly showed a parallel increase in presentations with both T1-T2 and T3-T4 disease between 2000 and 2010, supporting the notion of a lack of stage shift between these two periods (Figure 5).

Changes in grade presentation. We finally assessed for changes in the histological diagnosis rate of low, intermediate and highgrade tumours across the cohort. In analysis of the whole group, there was a striking shift in grade attribution over time. Low-grade cancers in particular were the most common type in the early years of the decade but intermediate-grade cancers became the predominant type in the latter half of the decade (Figure 6). In analysis of the two time periods, there was a significant reduction in the IR of low-grade cancers ( 91 vs $81, P<0.0001)$. In contrast, both intermediate- and high-grade cancers had apparently increased over the decade: intermediate-grade IR 65 vs 143 $(P<0.00001)$, high-grade IR 44 vs $72(P<0.00001$; Table 1$)$. We further assessed changes in histological grade based on analysis of the yearly data across different age groups (Figure 6). Here, we again observed a clear increase in intermediate-grade cancers across all age groups (Figure 6). High-grade cancers also increased across all age groups albeit at a slower rate (Figure 6). Low-grade cancer IR however remained stable or fell across the age groups (Figure 6). These data suggest that there has been a significant change in grade attribution of prostate cancer in the last decade. Notably, a relative reduction in the numbers of low-grade cancers diagnosed and a significant increase in the proportions of intermediate- and high-grade cancers diagnosed. Intermediategrade cancers now represent the majority of new histological diagnosis in the most contemporary series.

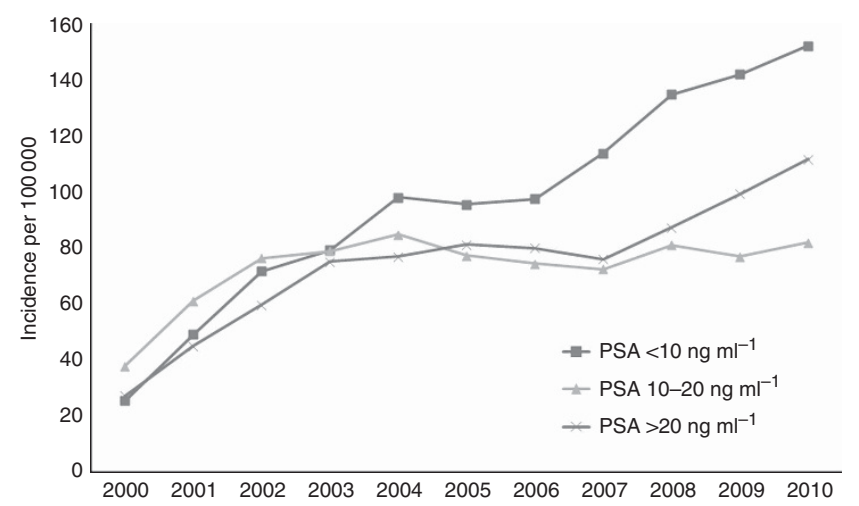

Figure 4. Incidence rate of presentation with different PSA ranges 2000-2010.

\section{DISCUSSION}

In a previous UK population study, Brewster et al (2000) identified increases in cancer IRs of nearly two-fold from 1981 to 1996. Initially, because of an increase in transurethral resection of the prostate for obstructive symptoms and later because of an increase in PSA testing. Data from the present study would suggest that this rate of increase in detection is being maintained and is in line with the projected increases over the next 20 years (Mistry et al, 2011).

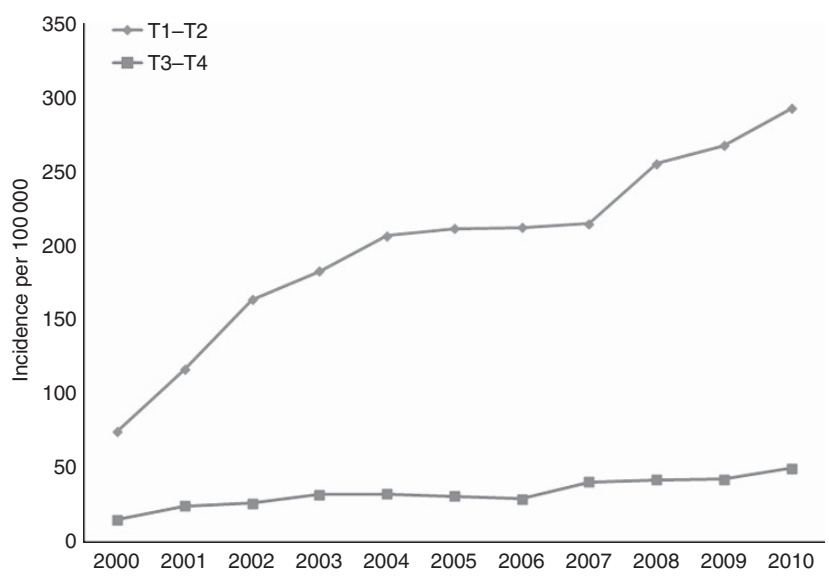

Figure 5. Incidence rate of presentation with either organ confined (T1-T2) or locally advanced (T3-T4) tumours 2000-2010.
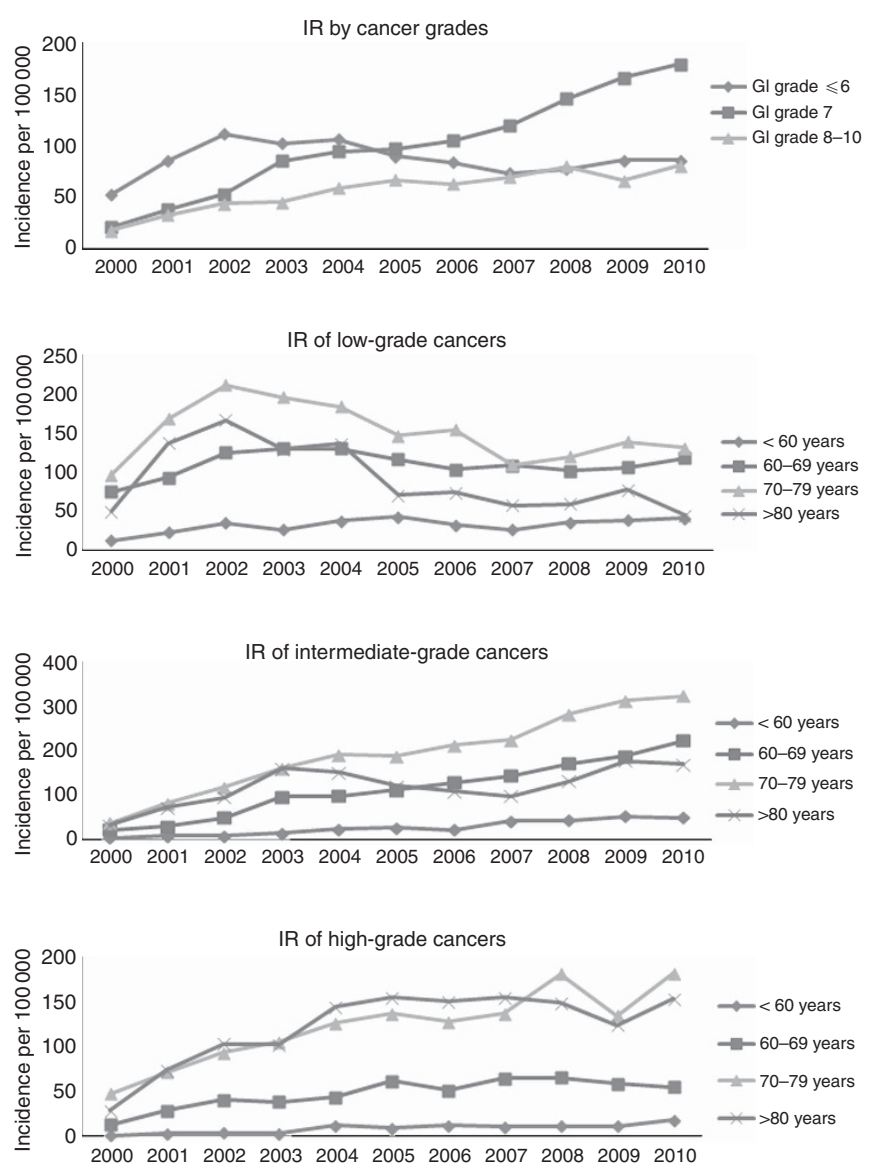

Figure 6. Incidence rates (IR) stratified by Gleason grade and by age group 2000-2010. 
The protect study has highlighted the even greater incidence of prostate cancer should a PSA-based screening test be introduced in the United Kingdom (Moore et al, 2009). In a comparative study with a similar population from the East of England, it was projected that PSA screening in men aged 50-69 years would increase the detection of prostate cancer from 130 to 2660 new diagnosis per 100000 population. The principal disadvantage of such an approach, however, would be over diagnosis of low-risk and indolent cancers (Moore et al, 2009). PSA-based screening for prostate cancer is therefore unlikely to be adopted in the United Kingdom in the near future.

The key factor in localised prostate cancer management is the risk category of the disease diagnosed. A recent study from two hospitals based in the East of England has reported that the majority of localised cancers detected from primary care referrals were of intermediate- or high-risk type (Serag et al, 2012). Indeed, this has now been verified in this present study where only a minority of contemporary cancers were categorised as low risk. What is striking, however, is that the IRs of men classified as having intermediate- and high-risk disease has been increasing, whereas that of low-risk disease has been relatively stable over the last 10 years. To our knowledge, this is the first study to report the observation of an upward shift in risk status in a UK populationbased study. A change in the TNM classification system (fifth edition to seventh edition) is unlikely to be the cause for this, as it was only introduced into the registry in 2010. This increase in intermediate- and high-risk disease has paradoxically occurred despite a steep increase in the relative proportions of men diagnosed at PSA levels of $<10 \mathrm{ng} \mathrm{ml}^{-1}$. Referrals from GPs are guided by the Department of Health guidelines for cancer referrals first formulated in 2000 and then further updated in 2005 (Department of Health. The NHS cancer plan; National Institute for Health and Clinical Excellence. Referral guidelines for suspected cancer. Clinical guideline 27). Importantly, however, the criteria for raised PSA referrals were the same in both versions (that is, age-specific PSA). Hence, there has not been a change in recommendations for PSA thresholds for referral. Local network guidelines similarly have not been significantly altered to our knowledge. We hypothesise therefore that the observed change in presenting PSA levels is probably due to a greater awareness of prostate cancer and uptake of opportunistic testing, which has driven both the overall rise in diagnostic rates and increased detection at lower PSA levels.

The primary reason for the observed change in risk-group IRs was a significant increase in the numbers of histological Gleason sum 7 cancers and a relative fall in Gleason sum 6 cancers. Another clear observation in this study was a relative fall in numbers of men presenting with metastatic disease, which is consistent with other recent population-based studies (Adolfsson et al, 2007). In these studies, however, a decrease in metastatic rates is commonly associated with an increase in diagnoses of Grade 6 and more organ-confined tumours. In contrast, in our study we have observed a paradoxical upward grade migration and no change in clinical stages despite falling metastasis rates. One other study has reported recent data on grade presentation in the United Kingdom. Shafique et al (2012) in an elegant study reported on the relationship between socioeconomic status and grade presentation in a Scottish population from 1991 to 2007. In that study, however, risk groups were not used and the low-grade definition included any tumour of Gleason score $\leqslant 7$, and hence it could not differentiate between Gleason sum 6 and 7 tumours.

The increase in grade calling in this report is unlikely to be due to a true increase in disease aggressiveness. Instead, it is most likely to be a result of a change in the pathological interpretation of diagnostic biopsies. Several reports from the United States have similarly found an apparent upward grade migration towards more intermediate and high-grade disease as a result of changes in reporting practice, although ours is the first to document this phenomenon in the United Kingdom (Derweesh et al, 2004; Jang et al, 2006; Falzarano and Magi-Galluzzi, 2011). This in turn may be in part due to the updating of the Gleason scoring system, which was reviewed in 2005 (Epstein et al, 2005). Indeed, in this study the two time periods selected (2000-2005 and 2006-2010) allow for a convenient comparison of histological interpretation before and after this review. Our results provide strong support for the notion that this review may have materially changed the histological classification of prostate cancers in the United Kingdom and resulted in upward grade migration. Ideally, we would have liked to review the pathology of slides before 2005 using current pathological criteria but this was outside the scope of the present cohort study. In a recent US paper, however, Dong et al (2012) did apply the revised scoring system to a historical cohort of Gleason sum 6 tumours from radical prostatectomy samples and found that $34 \%$ of patients were upgraded to Gleason sum 7 or 8 by the new criteria. Moreover, the reclassification more accurately predicted the subsequent behaviour of the tumours in terms of biochemical relapse and disease progression. These and other studies highlight the key importance of the pathologist in ascribing risk classification and its subsequent impact on deciding a patient's treatment as well as outcome. Ascribing an intermediate or high Gleason sum score will usually trigger a recommendation for active (radical) treatment as opposed to surveillance alone by clinicians and multidisciplinary teams managing men with prostate cancer.

An increase in the proportions of men diagnosed with disease eligible for active treatment has significant implications for the clinical provision of prostate cancer services. It is already recognised that the numbers of men receiving radical therapy for localised prostate cancer have increased dramatically in the United Kingdom (Vesey et al, 2012). If the trends in this study are reflected nationally, then this increase is not only driven by an overall increase in incidence but also by more cancers being considered eligible for therapy by virtue of being categorised as intermediate or high risk. The use of enhanced biopsy methods (for example, extended transrectal biopsies and template perineal biopsies) as well as advanced imaging (for example, functional MRI) is also increasing and the impact of this on diagnosis rates and disease presentation is unknown (Lawrence et al, 2012; Nelson et al, 2013). The rising costs of cancer care are increasingly being noted worldwide. In the United States, it is projected that in the next 10 years, cancer care costs will rise by $>25 \%$ with the largest increase in spending attributed to prostate cancer (Mariotto et al, 2011). Currently, the United Kingdom spends the least per patient on prostate cancer compared with other European counterpart countries (Fourcade et al, 2010). Given the trends observed in this study and the projected national increase in diagnosis rates, it is likely that the United Kingdom and NHS will need to see per capita spending on prostate cancer therapy increase dramatically in the future.

This report has a number of limitations inherent in a study reliant on cancer registry data. Most importantly, the data are based on the accuracy of the recorded information kept in the National Cancer Registration Service. There was no central review of histology and, as mentioned, stage data were based on a combination of radiological, clinical or pathological data. Of note, however, only a minority of men would have had pathology-based staging from radical prostatectomy. We cannot comment on any differences in symptomatic presentation for this cohort as these data are not collected routinely by the registry. However, we are not aware of any material change to the local referral guidelines with regards symptoms within the study time frame. Finally, we fully acknowledge that 1 of 28 cancer networks and as such our findings may not be reflective of national practice. However, we have no reason to suspect that the patterns observed in this region would be outliers in terms of the approach and methods of prostate cancer detection and diagnosis. 
In conclusion, this study confirms increasing rates of prostate cancer diagnosis in a UK population. Although presentation with metastatic disease is falling, we report for the first time that there is a paradoxical increase in the relative proportions of men classified as having intermediate- and high-risk localised cancers. We further observe that the critical contributory factor is likely to be a change in histological reporting of prostate cancer biopsies rather than a true change in diseases aggressiveness. However, further work is needed to explore this notion and to verify the observations in other cancer networks. In particular, we would propose a focused study looking at pathological interpretation of diagnostic biopsies before and after 2005 with cohort studies undertaking centralised histology review. Nevertheless, the current data suggest a significant change in the presenting disease profile of prostate cancer in the United Kingdom, and this will need considering when planning prostate cancer treatment resources especially in the context of a centrally funded national health system.

\section{ACKNOWLEDGEMENTS}

We are very grateful for the expert assistance of Dr C Brown and Dr B Rous in assigning tumour stage and the staff of the NCRS(E) for compiling the registration data.

\section{CONFLICT OF INTEREST}

The authors declare no conflicts of interest.

\section{AUTHOR CONTRIBUTIONS}

Data acquisition and collation was done by DAG and KW; analysis of data was performed by AL, KRM, DG and VJG; study concept and design was done by VJG; manuscript drafting and review was done by VJG, AL, KRM, DAG and KW.

\section{REFERENCES}

Adolfsson J, Garmo H, Varenhorst E, Ahlgren G, Ahlstrand C, Andrén O, BillAxelson A, Bratt O, Damber JE, Hellström K, Hellström M, Holmberg E, Holmberg L, Hugosson J, Johansson JE, Petterson B, Törnblom M, Widmark A, Stattin P (2007) Clinical characteristics and primary treatment of prostate cancer in Sweden between 1996 and 2005. Scand J Urol Nephrol 41(6): 456-477.

Brewster DH, Fraser LA, Harris V, Black RJ (2000) Rising incidence of prostate cancer in Scotland: increased risk or increased detection? BJU Int 85(4): 463-472.

Department of Health. The NHS cancer plan. London: Department of Health (2000) Department of Health, 'Delivering the Cancer Reform Strategy. Report by the Controller and Auditor General,' Tech. rep., HC 568., Session 2010-2011. Available at http://www.nao.org.uk/wp-content/ uploads/2010/11/1011568.pdf.

Derweesh IH, Kupelian PA, Zippe C, Levin HS, Brainard J, Magi-Galluzzi C, Myles J, Reuther AM, Klein EA (2004) Continuing trends in pathological stage migration in radical prostatectomy specimens. Urol Oncol 22(4): 300-306.
Dong F, Wang C, Farris AB, Wu S, Lee H, Olumi AF, McDougal WS, Young RH, Wu CL (2012) Impact on the clinical outcome of prostate cancer by the 2005 international society of urological pathology modified Gleason grading system. Am J Surg Pathol 36(6): 838-843.

Epstein JI, Allsbrook Jr WC, Amin MB, Egevad LL. ISUP Grading Committee (2005) The 2005 International Society of Urological Pathology (ISUP) Consensus Conference on Gleason Grading of Prostatic Carcinoma. Am J Surg Pathol 29(9): 1228-1242.

Falzarano SM, Magi-Galluzzi C (2011) Prostate cancer staging and grading at radical prostatectomy over time. Adv Anat Pathol 18(2): 159-164.

Fourcade RO, Benedict A, Black LK, Stokes ME, Alcaraz A, Castro R (2010) Treatment costs of prostate cancer in the first year after diagnosis: a short-term cost of illness study for France, Germany, Italy, Spain and the UK. BJU Int 105(1): 49-56.

Jang TL, Han M, Roehl KA, Hawkins SA, Catalona WJ (2006) More favorable tumor features and progression-free survival rates in a longitudinal prostate cancer screening study: PSA era and threshold-specific effects. Urology 67(2): 343-348.

Lawrence EM, Gnanapragasam VJ, Priest AN, Sala E (2012) The emerging role of diffusion-weighted MRI in prostate cancer management. Nat Rev Urol 9(2): 94-101.

Mariotto AB, Yabroff KR, Shao Y, Feuer EJ, Brown ML (2011) Projections of the cost of cancer care in the United States: 2010-2020. J Natl Cancer Inst 103(2): 117-128.

Mistry M, Parkin DM, Ahmad AS, Sasieni P (2011) Cancer incidence in the United Kingdom: projections to the year 2030. Br J Cancer 105(11): 1795-1803.

Moore AL, Dimitropoulou P, Lane A, Powell PH, Greenberg DC, Brown CH, Donovan JL, Hamdy FC, Martin RM, Neal DE (2009) Population-based prostate-specific antigen testing in the UK leads to a stage migration of prostate cancer. BJU Int 104(11): 1592-1598.

National Institute for Health and Clinical Excellence (2005) Referral guidelines for suspected cancer. Clinical guideline 27. London:National Institute for Health and Clinical Excellence.

Neal DE, Donovan JL, Martin RM, Hamdy FC (2009) Screening for prostate cancer remains controversial. Lancet 374(9700): 1482-1483.

Nelson AW, Harvey RC, Parker RA, Kastner C, Doble A, Gnanapragasam VJ (2013) Repeat prostate biopsy strategies after initial negative biopsy: meta-regression comparing cancer detection of transperineal, transrectal saturation and MRI guided biopsy. PLoS One 8(2): e57480.

Schröder FH, Hugosson J, Roobol MJ, Tammela TL, Ciatto S, Nelen V, Kwiatkowski M, Lujan M, Lilja H, Zappa M, Denis LJ, Recker F, Berenguer A, Määttänen L, Bangma CH, Aus G, Villers A, Rebillard X, van der Kwast T, Blijenberg BG, Moss SM, de Koning HJ, Auvinen A. ERSPC Investigators (2009) Screening and prostate-cancer mortality in a randomized European study. N Engl J Med 360(13): 1320-1328.

Serag H, Banerjee S, Saeb-Parsy K, Irving S, Wright K, Stearn S, Doble A, Gnanapragasam VJ (2012) Risk profiles of prostate cancers identified from UK primary care using national referral guidelines. Br J Cancer 106(3): 436-439.

Shafique K, Oliphant R, Morrison DS (2012) The impact of socio-economic circumstances on overall and grade-specific prostate cancer incidence: a population-based study. Br J Cancer 107(3): 575-582.

Vesey SG, McCabe JE, Hounsome L, Fowler S (2012) UK radical prostatectomy outcomes and surgeon case volume: based on an analysis of the British Association of Urological Surgeons Complex Operations Database. BJU Int 109(3): 346-354.

This work is published under the standard license to publish agreement. After 12 months the work will become freely available and the license terms will switch to a Creative Commons AttributionNonCommercial-Share Alike 3.0 Unported License. 